Harmonic interpolation based on Radon projections along the sides of regular polygons
I. Georgieva
C. Hofreither
C. Koutschan
V. Pillwein

T. Thanatipanonda

Supported by

Austrian Science Fund (FWF)

Upper Austria

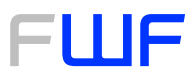




$\begin{array}{ll}\text { Editorial Board: } & \text { Bruno Buchberger } \\ & \text { Bert Jüttler } \\ & \text { Ulrich Langer } \\ & \text { Esther Klann } \\ & \text { Peter Paule } \\ & \text { Clemens Pechstein } \\ & \text { Veronika Pillwein } \\ & \text { Ronny Ramlau } \\ & \text { Josef Schicho } \\ & \text { Wolfgang Schreiner } \\ & \text { Franz Winkler } \\ & \text { Walter Zulehner }\end{array}$

Managing Editor: Veronika Pillwein

Communicated by: Ulrich Langer

Josef Schicho

DK sponsors:

- Johannes Kepler University Linz (JKU)

- Austrian Science Fund (FWF)

- Upper Austria 


\title{
Harmonic interpolation based on Radon projections along the sides of regular polygons
}

\author{
I. Georgieva* \\ C. Hofreither ${ }^{\dagger}$ \\ C. Koutschan ${ }^{\ddagger}$ \\ V. Pillwein ${ }^{\S}$ \\ T. Thanatipanonda
}

\begin{abstract}
Given information about a harmonic function in two variables, consisting of a finite number of values of its Radon projections, i.e., integrals along some chords of the unit circle, we study the problem of interpolating these data by a harmonic polynomial. With the help of symbolic summation techniques we show that this interpolation problem has a unique solution in the case when the chords form a regular polygon. Numerical experiments for this and more general cases are presented.
\end{abstract}

\section{Introduction}

The Radon transform, studied by Johann Radon in the early twentieth century, is the theoretical foundation for tomography methods for shape reconstruction of objects with non-homogeneous density. These methods were intensively studied in the 1960s and continue to find many applications in medicine, electronic microscopy, geology, plasma investigations, finding defects in nuclear reactors, etc. From the mathematical point of view, the problem is to recover a multivariate function using information based on line integrals of the unknown function. Among the difficulties the recovery methods have to face are: huge amount of data necessary to achieve high accuracy; impossibility to get enough data due to technical reasons; incomplete projection data; measurement noise in the given data. The employed methods may be loosely grouped into integral and algebraic type. The integral methods are based on the inverse Radon transform. Here all considerations are in continuous form and they come to discretization immediately before the implementation of the recovery algorithm. In the algebraic methods, discretization of the problem is carried out immediately, and the problem is then reduced to solving a linear or nonlinear system of equations.

\footnotetext{
*Institute of Mathematics and Informatics, Bulgarian Academy of Sciences, Acad. G. Bonchev, Bl. 8, 1113, Sofia, Bulgaria, irina@math.bas.bg

${ }^{\dagger}$ Doctoral College Computational Mathematics, Johannes Kepler University, Altenberger Str. 69, 4040 Linz, Austria, clemens.hofreither@dk-compmath.jku.at

${ }^{\ddagger}$ Research Institute for Symbolic Computation (RISC), Johannes Kepler University, Altenberger Str. 69, 4040 Linz, Austria, Koutschan@risc.jku.at

${ }^{\S}$ Research Institute for Symbolic Computation (RISC), Johannes Kepler University, Altenberger Str. 69, 4040 Linz, Austria, veronika.pillwein@risc.jku.at

${ }^{\top}$ Research Institute for Symbolic Computation (RISC), Johannes Kepler University, Altenberger Str. 69, 4040 Linz, Austria, thotsaporn@gmail.com
} 
In the present work, we consider an algebraic method where the solution is sought by means of an interpolation problem. More precisely, for given values of some Radon projections, we seek a polynomial function which matches these data exactly.

An idea suggested by B. Bojanov is to incorporate additional knowledge about the function to be recovered into approximation methods. It is to be expected that this can improve the accuracy of the approximation while reducing the amount of input data required as well as the computational effort. In applications, such problem-specific knowledge is often provided in the form of a partial differential equation which the unknown satisfies.

In the present work, we concern ourselves with the simple case when the unknown is harmonic, i.e., satisfies the Laplace equation $\Delta u=0$. This elliptic partial differential equation is important both as a model problem as well as in actual applications, like heat transport, diffusion problems or Stokes flow of incompressible fluids.

One natural tool for approximating harmonic functions are harmonic polynomials. These polynomials belong to the class of holonomic functions, a class for which over the past decades several symbolic algorithms have been developed to deal with, e.g., problems of symbolic integration and/or summation. We use symbolic techniques, such as creative telescoping and recurrence solving, to derive a closed form for Radon projections of the harmonic basis functions. We then use this result to derive a class of regular interpolation schemes for harmonic functions with Radon projections as given data.

\section{Preliminaries and related work}

Let $I(\theta, t)$ denote a chord of the unit circle at angle $\theta \in[0,2 \pi)$ and distance $t \in(-1,1)$ from the origin (see Figure 1). The chord $I(\theta, t)$ is parameterized by

$$
s \mapsto(t \cos \theta-s \sin \theta, t \sin \theta+s \cos \theta)^{\top}, \quad \text { where } s \in\left(-\sqrt{1-t^{2}}, \sqrt{1-t^{2}}\right) .
$$

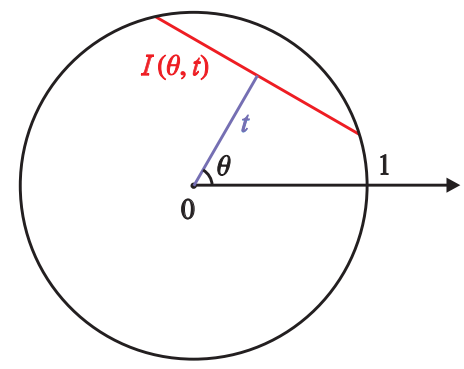

Figure 1: The chord $I(\theta, t)$ of the unit circle.

Definition 1. Let $f(x, y)$ be a real-valued bivariate function in the unit disk in $\mathbb{R}^{2}$. The Radon projection $\mathcal{R}_{\theta}(f ; t)$ of $f$ in direction $\theta$ is defined by the line integral

$$
\mathcal{R}_{\theta}(f ; t):=\int_{I(\theta, t)} f(\mathbf{x}) d \mathbf{x}=\int_{-\sqrt{1-t^{2}}}^{\sqrt{1-t^{2}}} f(t \cos \theta-s \sin \theta, t \sin \theta+s \cos \theta) d s .
$$

Johann Radon [24] showed in 1917 that a differentiable function $f$ is uniquely determined by the values of its Radon transform,

$$
f \mapsto\left\{\mathcal{R}_{\theta}(f ; t):-1 \leq t \leq 1,0 \leq \theta<\pi\right\} .
$$


In the following we formulate the problem of recovery of a polynomial from a finite number of values of its Radon transform. Essentially, this may be viewed as a bivariate interpolation problem where the traditional point values are replaced by the means over chords of the unit circle.

Let $\Pi_{n}^{2}=\left\{\sum_{i+j \leq n} \alpha_{i j} x^{i} y^{j}: \alpha_{i j} \in \mathbb{R}\right\}$ denote the space of real bivariate polynomials of total degree at most $n$. This space has dimension $\left(\begin{array}{c}n+2 \\ 2\end{array}\right)$. Assume that a set $\mathcal{I}=$ $\left\{I_{m}=I\left(\theta_{m}, t_{m}\right): m=1, \ldots,\left(\begin{array}{c}n+2 \\ 2\end{array}\right)\right\}$ of chords of the unit circle is given. Furthermore, to each chord $I \in \mathcal{I}$ a given value $\gamma_{I} \in \mathbb{R}$ is associated. Then, the aim is to find a polynomial $p \in \Pi_{n}^{2}$ such that

$$
\int_{I} p(\mathbf{x}) d \mathbf{x}=\gamma_{I} \quad \forall I \in \mathcal{I} .
$$

If this interpolation problem has a unique solution for every choice of values $\left\{\gamma_{I}\right\}$, then the scheme $\mathcal{I}$ of chords is called regular. The question of how to construct such regular schemes has been extensively studied. The first general result was given by Marr [20] in 1974, who proved that the set of chords connecting $n+2$ equally spaced points on the unit circle is regular for $\Pi_{n}^{2}$. A more general result for $\mathbb{R}^{d}$ and general convex domains was published by Hakopian [14] in 1982. Applied to the unit disk in $\mathbb{R}^{2}$, it states that even the chords connecting any $n+2$ distinct points on the unit circle form a regular scheme for $\Pi_{n}^{2}$.

Another family of regular schemes was provided by Bojanov and Georgieva [2]. They showed that a scheme consisting of $\left(\begin{array}{c}n+2 \\ 2\end{array}\right)$ chords partitioned into $n+1$ subsets such that the $k$-th subset consists of $k$ parallel chords is regular for $\Pi_{n}^{2}$, provided that the distances $t$ satisfy some additional conditions. Particular choices of suitable distances $t$ were later given by Georgieva and Ismail [10] in terms of zeroes of Chebyshev polynomials of the second kind, as well as Georgieva and Uluchev [11] in terms of zeroes of Jacobi polynomials.

Bojanov and $\mathrm{Xu}[5]$ proposed a regular scheme consisting of $\left(\begin{array}{c}n+2 \\ 2\end{array}\right)$ chords partitioned into $2\lfloor(n+1) / 2\rfloor+1$ equally spaced directions, such that in every direction there are $\lfloor n / 2\rfloor+1$ parallel chords. The distances $t$ of the chords are zeroes of Chebyshev polynomials of the second kind.

A mixed regular scheme which incorporates Radon projections and point evaluations on the unit circle was given by Georgieva, Hofreither, and Uluchev [9].

Many other mathematicians have worked on problems with applications in the mathematical foundations of computer tomography, among them $[16,6,7,8,15,19,21]$. Recovery of polynomials in two variables based on Radon projections is also considered in $[1,22,3,4,12,13]$.

\section{Interpolation by harmonic polynomials}

Assume that we know a priori that the function to be interpolated is harmonic. Then it seems natural to work in the space $\mathcal{H}_{n}$ of real bivariate harmonic polynomials of total degree at most $n$, which has dimension $2 n+1$. Analogous to (2), we prescribe chords $\mathcal{I}=\left\{I_{1}, I_{2}, \ldots, I_{2 n+1}\right\}$ of the unit circle and associated given values $\left\{\gamma_{I}\right\}$, and wish to find a harmonic polynomial $p \in \mathcal{H}_{n}$ such that

$$
\int_{I} p(\mathbf{x}) d \mathbf{x}=\gamma_{I} \quad \forall I \in \mathcal{I} .
$$


Again we call $\mathcal{I}$ regular if the interpolation problem (3) has a unique solution for all given values $\left\{\gamma_{I}\right\}$. In the following, we show how to construct one class of such regular schemes.

\subsection{Harmonic basis}

We use the basis of the harmonic polynomials

$$
\phi_{0}(x, y)=1, \quad \phi_{k, 1}(x, y)=\operatorname{Re}(x+\mathbf{i} y)^{k}, \quad \phi_{k, 2}(x, y)=\operatorname{Im}(x+\mathbf{i} y)^{k} .
$$

An expansion of the polynomial $p \in \mathcal{H}_{n}$ is then given by

$$
p(x, y)=p_{0}+\sum_{k=1}^{n} p_{k, 1} \phi_{k, 1}(x, y)+\sum_{k=1}^{n} p_{k, 2} \phi_{k, 2}(x, y),
$$

with the coefficient vector $\underline{p}=\left(p_{0}, p_{1,1}, p_{1,2}, \ldots, p_{n, 1}, p_{n, 2}\right)^{T} \in \mathbb{R}^{2 n+1}$. The interpolation problem (3) results then in the system of linear equations

$$
A \underline{p}=\underline{\gamma}
$$

with

$$
A=\left(\begin{array}{cccccc}
\int_{I_{1}} 1 & \int_{I_{1}} \phi_{1,1} & \int_{I_{1}} \phi_{1,2} & \cdots & \int_{I_{1}} \phi_{n, 1} & \int_{I_{1}} \phi_{n, 2} \\
\int_{I_{2}} 1 & \int_{I_{2}} \phi_{1,1} & \int_{I_{2}} \phi_{1,2} & \cdots & \int_{I_{2}} \phi_{n, 1} & \int_{I_{2}} \phi_{n, 2} \\
\vdots & \vdots & \vdots & \ddots & \vdots & \vdots \\
\int_{I_{2 n+1}} 1 & \int_{I_{2 n+1}} \phi_{1,1} & \int_{I_{2 n+1}} \phi_{1,2} & \cdots & \int_{I_{2 n+1}} \phi_{n, 1} & \int_{I_{2 n+1}} \phi_{n, 2}
\end{array}\right)
$$

The question whether the interpolation problem has a unique solution is thus equivalent to the question whether the matrix $A$ has non-zero determinant.

Using the binomial theorem, it is easy to obtain the representations

$$
\begin{aligned}
\phi_{k, 1}(x, y) & =\sum_{\ell=0}^{\lfloor k / 2\rfloor}\left(\begin{array}{c}
k \\
2 \ell
\end{array}\right)(-1)^{\ell} x^{k-2 \ell} y^{2 \ell}, \\
\phi_{k, 2}(x, y) & =\sum_{\ell=0}^{\lfloor k / 2\rfloor}\left(\begin{array}{c}
k \\
2 \ell+1
\end{array}\right)(-1)^{\ell} x^{k-(2 \ell+1)} y^{2 \ell+1},
\end{aligned}
$$

which give an expansion of the harmonic basis in terms of the monomial basis $x^{i} y^{j}$ of the polynomials. For Radon projections of monomials, we can obtain the following formula.

Lemma 1. For arbitrary $i, j \in \mathbb{N}_{0}$ and an arbitrary chord $I(\theta, t)$ of the unit disk with angle $0 \leq \theta<2 \pi$ and distance to the origin $t \in(-1,1)$, we have

$$
\begin{aligned}
\int_{I(\theta, t)} x^{i} y^{j} d \mathbf{x}=\sum_{p=0}^{i} \sum_{q=0}^{j} & \left(\begin{array}{l}
i \\
p
\end{array}\right)\left(\begin{array}{l}
j \\
q
\end{array}\right) t^{p+q}(\cos \theta)^{j+p-q}(\sin \theta)^{i-(p-q)} \times \\
& \times \frac{(-1)^{i-p}}{i+j-p-q+1}\left(1-t^{2}\right)^{\frac{1}{2}(i+j-p-q+1)}\left(1-(-1)^{i+j-p-q+1}\right) .
\end{aligned}
$$


Proof. Making use of the parameterization (1), we get

$$
\int_{I(\theta, t)} x^{i} y^{j} d \mathbf{x}=\int_{-\sqrt{1-t^{2}}}^{\sqrt{1-t^{2}}}(t \cos \theta-s \sin \theta)^{i}(t \sin \theta+s \cos \theta)^{j} d s .
$$

The statement follows by applying the binomial theorem and simple integration.

Making use of the linearity of the Radon transform, we can combine the above formula with (5) and (6) to get, for $k \geq 1$,

$$
\begin{aligned}
\int_{I(\theta, t)} \phi_{k, 1}= & \sum_{\ell=0}^{\lfloor k / 2\rfloor}\left(\begin{array}{c}
k \\
2 \ell
\end{array}\right)(-1)^{\ell} \sum_{p=0}^{k-2 \ell} \sum_{q=0}^{2 \ell}\left(\begin{array}{c}
k-2 \ell \\
p
\end{array}\right)\left(\begin{array}{c}
2 \ell \\
q
\end{array}\right) t^{p+q}(\cos \theta)^{2 \ell+p-q}(\sin \theta)^{k-2 \ell-(p-q)} \\
& \times \frac{(-1)^{k-2 \ell-p}}{k-p-q+1}\left(1-t^{2}\right)^{\frac{1}{2}(k-p-q+1)}\left(1-(-1)^{k-p-q+1}\right)
\end{aligned}
$$

and

$$
\begin{aligned}
& \int_{I(\theta, t)} \phi_{k, 2}=\sum_{\ell=0}^{\lfloor k / 2\rfloor}\left(\begin{array}{c}
k \\
2 \ell+1
\end{array}\right)(-1)^{\ell} \sum_{p=0}^{k-2 \ell-1} \sum_{q=0}^{2 \ell+1}\left(\begin{array}{c}
k-2 \ell-1 \\
p
\end{array}\right)\left(\begin{array}{c}
2 \ell+1 \\
q
\end{array}\right) \\
& \times t^{p+q}(\cos \theta)^{2 \ell+1+p-q}(\sin \theta)^{k-2 \ell-1-(p-q)} \\
& \times \frac{(-1)^{k-2 \ell-1-p}}{k-p-q+1}\left(1-t^{2}\right)^{\frac{1}{2}(k-p-q+1)}\left(1-(-1)^{k-p-q+1}\right) .
\end{aligned}
$$

In this work, for the most part, we restrict ourselves to the case where the chords $\mathcal{I}$ form a regular $(2 n+1)$-sided convex polygon inscribed in the unit circle (cf. Figure 2, first picture), i.e., $I_{m}=I\left(\theta_{m}, t_{m}\right)$ with

$$
\theta_{m}=\frac{2 \pi m}{2 n+1}, \quad t_{m}=t=\cos \frac{\pi}{2 n+1} \quad \text { for } m=1, \ldots, 2 n+1 .
$$

\section{Symbolic Simplifications}

We are now going to evaluate the complicated integrals, respectively sums (7) and (8). While it may be a tedious job to simplify these expressions by hand, we employ computer algebra methods which can be of great help in such tasks. These algorithms are designed to work on the class of holonomic functions [25]; in short, these are functions and sequences which satisfy "sufficiently many" linear differential equations and recurrence equations. These equations, together with some initial values, are used as a data structure to represent such functions. The set of holonomic functions is closed under many operations - such as addition, multiplication, certain substitutions, definite summation and integration - which can be executed algorithmically. Thus the strategy for tackling expressions (7) and (8) is to use the above mentioned closure properties to derive a difference-differential equation

for the whole expression. Together with some initial values this may even give rise to a closed-form solution. A more detailed introduction into the topic is given in [17], here we only outline the basic ideas with a simple example. Consider the expression

$$
f_{n}(t)=\sum_{k=0}^{2 n+1} s_{n, k}(t)=\sum_{k=0}^{2 n+1}\left(\begin{array}{c}
2 n+1 \\
k
\end{array}\right) k^{2} t^{k}
$$


For the following computations we make use of the Mathematica package HolonomicFunctions ${ }^{1}[18]$ that has been developed by the third-named author. The defining difference equations of the summand can be determined entirely automatically using this package with the Annihilator command:

$\operatorname{In}[1]:=$ Annihilator[Binomial $\left.[2 n+1, k] k^{2} t^{k},\{S[k], S[n]\}\right]$

Out[1] $=\left\{(k-2 n-3)(k-2 n-2) S_{n}-2(n+1)(2 n+3), k^{2} S_{k}+(k+1) t(k-2 n-1)\right\}$

Here $S[\mu]$ and, in the output, $S_{\mu}$, denote the forward shift in the variable $\mu$, i.e., $S_{\mu} a(\mu)=a(\mu+1)$. The defining equations for the summand are relatively easy to obtain since it is composed of very basic objects, like powers, binomials, and polynomials. The same holds for the input (7) that we are interested in. Starting from the defining difference equations for $s_{n, k}(t)$ a recurrence relation for the sum $f_{n}(t)$ can be computed by the method of creative telescoping which was proposed by Zeilberger [26]. This method constructs a recurrence for the summand which is of a special form, namely it consists of two parts with the following properties. The first part contains only shifts in the main variable $n$ and coefficients that are independent of the summation variable $k$. The second part is of the form $\left(S_{k}-1\right) \cdot Q \cdot s_{n, k}(t)$, where $Q$ is an operator with shifts in both $n$ and $k$ and rational function coefficients in $n, k$ and $t$. Hence, in our example this relation may take the form

$$
\begin{aligned}
& (2 n+1)(2 n t+t+1) s_{n+1, k}(t)-(2 n+3)(1+t)^{2}(2 n t+3 t+1) s_{n, k}(t) \\
& \quad+\left(S_{k}-1\right) \cdot\left(c_{1}(n, k, t) s_{n, k}(t)+c_{2}(n, k, t) s_{n, k+1}(t)+c_{3}(n, k, t) s_{n+1, k}(t)\right)=0 .
\end{aligned}
$$

Since in this example the quotients $s_{n, k+1}(t) / s_{n, k}(t)$ and $s_{n+1, k}(t) / s_{n, k}(t)$ are rational functions, we can actually get $c_{2}=c_{3}=0$ here. Summing over this difference equation with respect to $k$ yields a recurrence relation for $f_{n}(t)$ from the first part of the operator. The second part is easily evaluated by telescoping, i.e., by

$$
\sum_{k=k_{0}}^{k_{1}}(a(k+1)-a(k))=a\left(k_{1}+1\right)-a\left(k_{0}\right) .
$$

In many cases in practice this part simply telescopes to zero as in our example. Thus we end up with the following recurrence and initial value:

$$
(2 n+1)(2 n t+t+1) f_{n+1}(t)-(2 n+3)(t+1)^{2}(2 n t+3 t+1) f_{n}(t)=0, \quad f_{0}(t)=t .
$$

Using HolonomicFunctions this recurrence could also be obtained by merely plugging in the sum symbolically in Mathematica notation, i.e.,

$\ln [2]:=$ Annihilator[Sum[Binomial $\left.\left.[2 n+1, k] k^{2} t^{k},\{k, 0,2 n+1\}\right],\{S[n]\}\right]$

Out[2] $=\left\{(2 n+1)(2 n t+t+1) S_{n}-(2 n+3)(t+1)^{2}(2 n t+3 t+1)\right\}$

Having a recurrence relation at hand it might even be possible to derive a closed form solution using a recurrence solver. In our example the solution is easily determined to be

$$
f_{n}(t)=(2 n+1)(2 n t+t+1) t(1+t)^{2 n-1},
$$

\footnotetext{
${ }^{1}$ freely available for download at http://www.risc.jku.at/research/combinat/software/HolonomicFunctions /
} 
under appropriate assumptions on the range of $t$. This algorithm can be extended to multiple sums (such as (7) and (8)) and integrals, but still proceeds by the same ideas that were demonstrated above (for doing symbolic integration, the shifts need to be replaced by partial derivatives).

Coming back to the integral over $\phi_{k, 1}$ expanded as a triple sum in (7): the defining equations for the summand are again easy to obtain since it is composed of very basic objects, like powers, binomials, and polynomials. Applying the method of creative telescoping as outlined above delivers the following recurrence relation for the integrals:

$$
\begin{array}{r}
(k+5) F(k+4)-4 t \cos (\theta)(k+4) F(k+3)+2(k+3)\left(2 \cos ^{2}(\theta)+2 t^{2}-1\right) F(k+2) \\
-4 t \cos (\theta)(k+2) F(k+1)+(k+1) F(k)=0,
\end{array}
$$

where $F(k)=\int_{I(\theta, t)} \phi_{k, 1}$. Similarly it is found that $\int_{I(\theta, t)} \phi_{k, 2}$ satisfies the same recurrence (with different initial values). Since our software can deal with integrations equally well, we can also evaluate the definite integral that is obtained from the parametrization (1) and by plugging in the representations (5) and (6); the result is exactly the same fourth-order recurrence that is displayed above.

Next, Petkovšek's algorithm Hyper [23] is used to compute a basis of hypergeometric solutions to this recurrence. Since its order is 4 , there are at most four such solutions, but it is very likely that some or even all solutions are not hypergeometric, in which case the algorithm returns fewer solutions. However, in our examples we are lucky and obtain all four solutions, one of which is

$$
\frac{1}{k+1}\left(t \cos (\theta)+\sqrt{\sin ^{2}(\theta)\left(t^{2}-1\right)}+\sqrt{\cos ^{2}(\theta)\left(2 t^{2}-1\right)-t^{2}+2 t \cos (\theta) \sqrt{\sin ^{2}(\theta)\left(t^{2}-1\right)}}\right)^{k-1}
$$

(the others differ by signs only).

Comparing initial values yields (quite complicated) closed-form representations of the integrals (7) and (8), which are linear combinations of the four basis solutions. Further algebraic simplifications, using the assumption of a regular convex polygon (9), lead to the following evaluation of the matrix entries.

Lemma 2. For the case when the chords $\mathcal{I}$ form a regular convex polygon, see (9), the matrix entries admit the closed form

$$
\int_{I_{m}} \phi_{0}=\alpha_{0}, \quad \int_{I_{m}} \phi_{k, 1}=\alpha_{k} \cos \left(k \theta_{m}\right), \quad \int_{I_{m}} \phi_{k, 2}=\alpha_{k} \sin \left(k \theta_{m}\right),
$$

where

$$
\alpha_{k}=\frac{2}{k+1} \sin \frac{(k+1) \pi}{2 n+1}>0 \quad \text { for } k=0, \ldots, n
$$

Proof. We have already outlined above how the result of this lemma can be obtained (and proven!) by computer algebra methods. The drawback of such methods is that often the intermediate results and the certificates which constitute the proof are way too large to be printed in a paper. However, the interested reader can find the details of our proof in the supplementary electronic material ${ }^{2}$ which is given as a Mathematica notebook.

\footnotetext{
${ }^{2}$ http://www.risc.jku.at/people/ckoutsch/material/RadonProjectionsElectronicMaterial.nb
} 


\section{Main result}

Theorem 3. Let the chords $\mathcal{I}$ form a regular convex polygon as in (9). Then the interpolation problem (3) has a unique solution in $\mathcal{H}_{n}$ for any given data $\underline{\gamma}$.

Proof. Lemma 2 gives a closed form for the matrix entries in the system of linear equations (4). Using linearity of the determinant in every column, we take out the factors $\alpha_{k}$ and get

$$
\operatorname{det} A=\alpha_{0} \prod_{k=1}^{n} \alpha_{k}^{2} \operatorname{det} B
$$

with

$$
B=\left(\begin{array}{cccccc}
1 & \cos \left(\theta_{1}\right) & \sin \left(\theta_{1}\right) & \ldots & \cos \left(n \theta_{1}\right) & \sin \left(n \theta_{1}\right) \\
1 & \cos \left(\theta_{2}\right) & \sin \left(\theta_{2}\right) & \ldots & \cos \left(n \theta_{2}\right) & \sin \left(n \theta_{2}\right) \\
\vdots & \vdots & \vdots & \ddots & \vdots & \vdots \\
1 & \cos \left(\theta_{2 n}\right) & \sin \left(\theta_{2 n}\right) & \ldots & \cos \left(n \theta_{2 n}\right) & \sin \left(n \theta_{2 n}\right) \\
1 & \cos \left(\theta_{2 n+1}\right) & \sin \left(\theta_{2 n+1}\right) & \ldots & \cos \left(n \theta_{2 n+1}\right) & \sin \left(n \theta_{2 n+1}\right)
\end{array}\right)
$$

The columns of $B$ consist of evaluations of the functions $1, \cos (x), \sin (x), \cos (2 x), \sin (2 x)$, $\ldots, \cos (n x), \sin (n x)$ at the points $\theta_{1}, \ldots, \theta_{2 n+1}$. These functions form a basis of the trigonometric polynomials of degree at most $n$. It is a classical result that $\operatorname{det} B \neq 0$ for distinct $0 \leq \theta_{1}<\theta_{2}<\ldots<\theta_{2 n+1}<2 \pi$, which concludes the proof together with $\alpha_{k}>0 \forall k$.

Remark. In the proof of Lemma 2, a very specific choice for the $\theta_{m}$ and $t_{m}$ was assumed. However, it can be shown that the result holds for general angles, $0 \leq \theta_{1}<\theta_{2}<\ldots<$ $\theta_{2 n+1}<2 \pi$, of the chords while the distances $t_{m}=t$ to the origin are constant and $t$ is not a zero of any Chebyshev polynomial $U_{1}, \ldots, U_{n}$. An analytic proof of this more general result will be the subject of a forthcoming paper. Some regular schemes according to this generalization are shown in Figure 2, and numerical results using such schemes are given in Section 6.
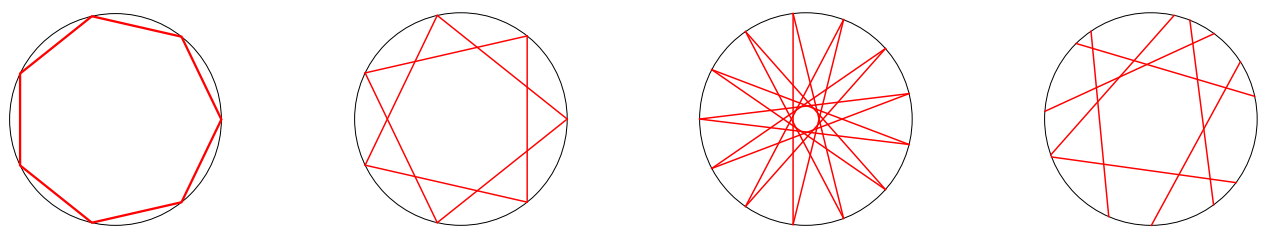

Figure 2: Regular schemes according to Remark 5.

\section{Numerical examples}

\subsection{Example 1}

We approximate the harmonic function

$$
u(x, y)=\exp (x) \cos (y)
$$



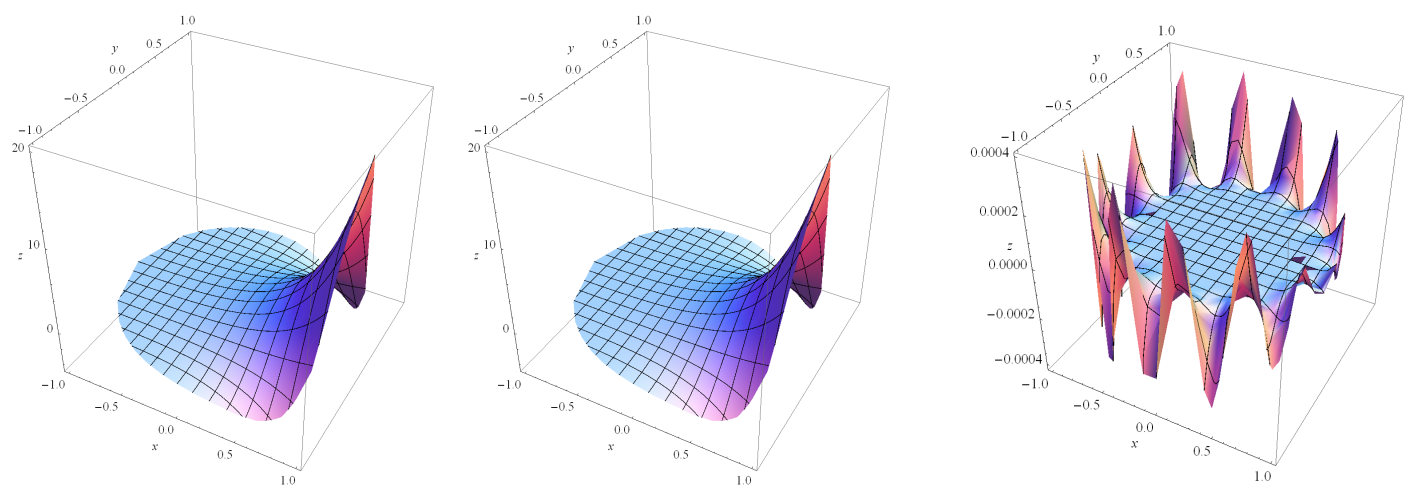

Figure 3: Example 1, $n=12$ : function $u$, interpolant $p$, error $u-p$

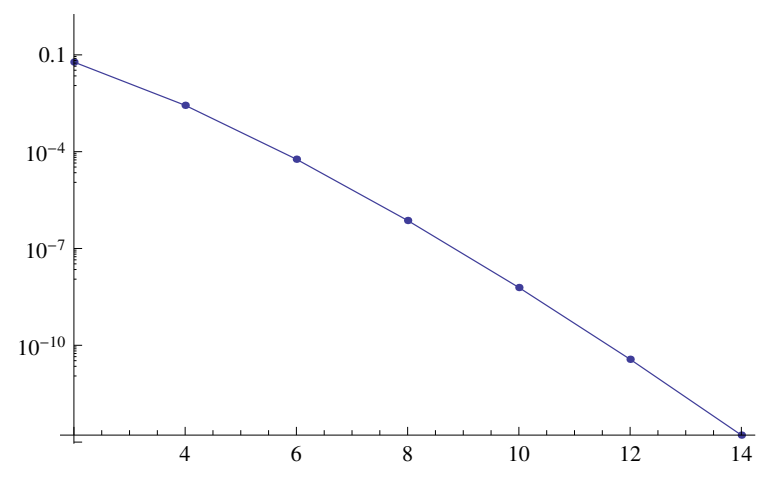

Figure 4: Example 1: errors. x-axis: degree of interpolating polynomial. y-axis: relative $L_{2}$-error

by a harmonic polynomial $p \in \mathcal{H}_{n}$ given $2 n+1$ values of its Radon projections taken along the edges of a regular $(2 n+1)$-sided convex polygon (Figure 2, first picture), i.e., $\theta_{m}$ and $t_{m}$ are chosen according to (9). In Figure 3, we display the function $u$ as well as its interpolating polynomial of degree 12 (using information from 25 chords) and the resulting error. For Figure 4, we vary the degree of the interpolating polynomial and plot the resulting relative $L_{2}$-errors. We see that the error decreases exponentially with $n$, indicating that the smooth function $u$ is being approximated with optimal order.

\subsection{Example 2}

We approximate the harmonic function

$$
u(x, y)=\log \sqrt{(x-1)^{2}+(y-1)^{2}}
$$

by a harmonic polynomial $p \in \mathcal{H}_{n}$ given the Radon projections along two families of chords: first, using a regular $(2 n+1)$-sided convex polygon as in the previous example, and second, using a star-like scheme as shown in Figure 2, second and third picture. The latter family of chords is constructed using the same equidistant points on the unit circle, 

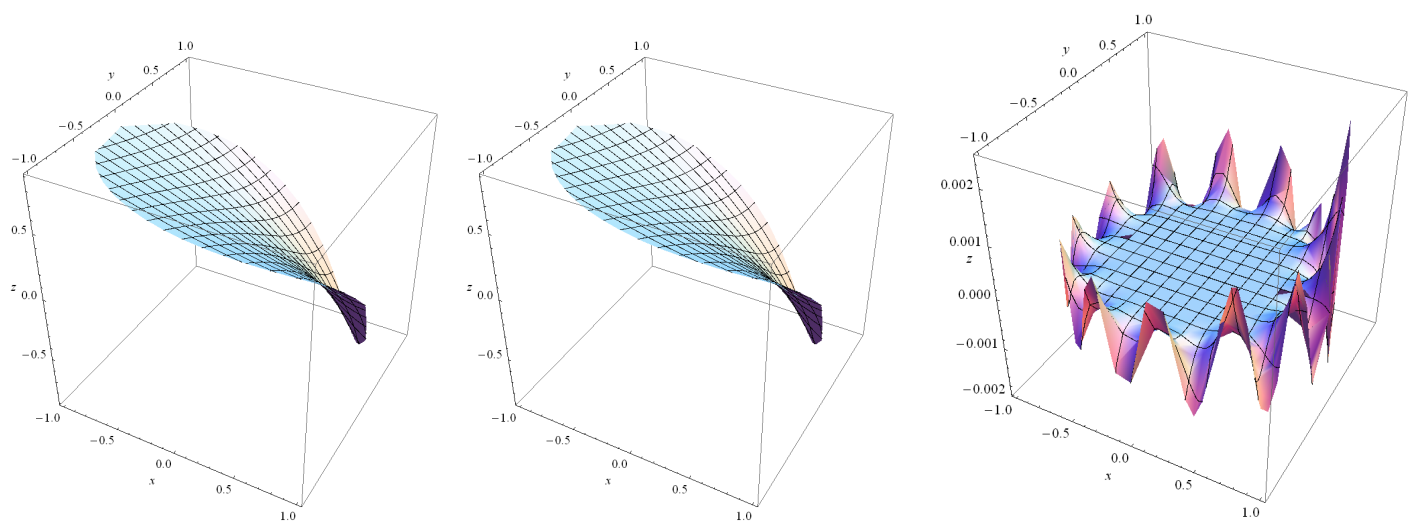

Figure 5: Example 2, $n=12$ : function $u$, interpolant $p$, error $u-p$

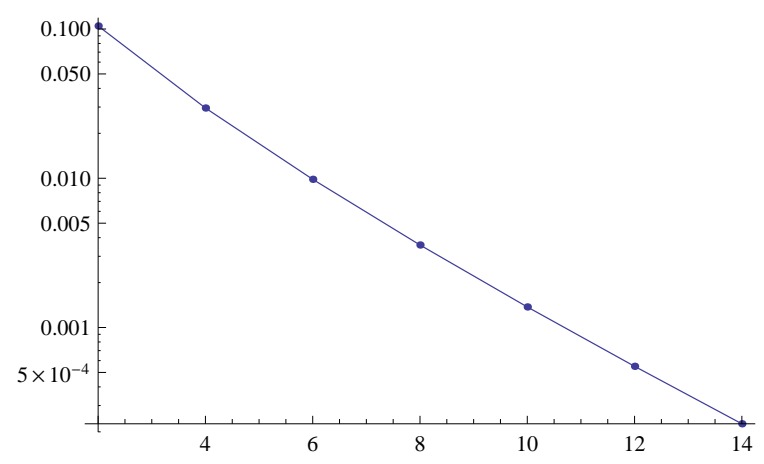

Figure 6: Example 2: errors. x-axis: degree of interpolating polynomial. y-axis: relative $L_{2}$-error

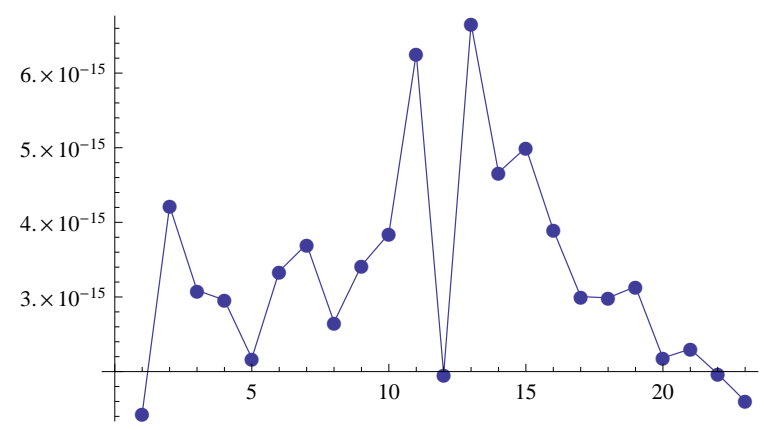

Figure 7: Example 2: relative $L_{2}$-error for regular stars with $\ell=1, \ldots, 23$ (47 chords). 
say $\left\{x_{1}, \ldots, x_{2 n+1}\right\}$, but now instead of joining the pairs of points $\left(x_{m}, x_{m+1}\right)$ for any $m$, we join the pairs of points $\left(x_{m}, x_{m+\ell}\right)$ for some $\ell \in \mathbb{N}$. Here indices which exceed $2 n+1$ are implicitly assumed to wrap back into the range $\{1, \ldots, 2 n+1\}$. Note that when $\ell=1$, we again get a regular convex polygon (Figure 2 , first picture), which corresponds to the shortest possible chords given the points $\left\{x_{1}, \ldots, x_{2 n+1}\right\}$, while the choice $\ell=n$ joins points which are "almost opposite" of each other, creating the longest possible chords (Figure 2, third picture).

In terms of the angles and distances of the chords, $I_{m}=I\left(\theta_{m}, t_{m}\right)$, such a star is described by the formulae

$$
\theta_{m}=\frac{\pi(2 m+\ell-1)}{2 n+1}, \quad t_{m}=t=\cos \frac{\ell \pi}{2 n+1} \quad \text { for } m=1, \ldots, 2 n+1,
$$

which simplify to (9) for the choice $\ell=1$. In order to ensure that $t$ is not a zero of any of $U_{1}, \ldots, U_{n}$ (as required by Remark 5), it is necessary and sufficient for $2 n+1$ and $\ell$ to be relatively prime.

In Figure 5, we display the function $u$ as well as its interpolating polynomial of degree 12 (using information from 25 chords) for the case $\ell=1$, i.e., the convex polygon case, and the resulting error. For varying interpolation degree $n$, we plot the relative $L_{2}$-errors in Figure 6. Again, convergence is exponential in $n$, although the errors are now larger as compared to Example 1 due to the singularity of $u$ close to the unit disk.

In order to compare the results for different star schemes with the same degree of interpolating polynomial, we choose now $n=23$, such that $2 n+1$ is a prime number and thus all stars $\ell=1, \ldots, n$ are admissible. The relative $L_{2}$-errors for $\ell=1, \ldots, n$ are plotted in Figure 7. The largest and smallest errors appear to be of the same order, differing at most by a factor of approximately 4 .

\subsection{Example 3}

In order to study the behavior of the method for functions with less smoothness, we construct the harmonic extension of the boundary function $g(\theta)=\theta^{2}$ on the unit circle in radial coordinates, where the argument $\theta$ is chosen in the interval $[-\pi, \pi]$. This function is
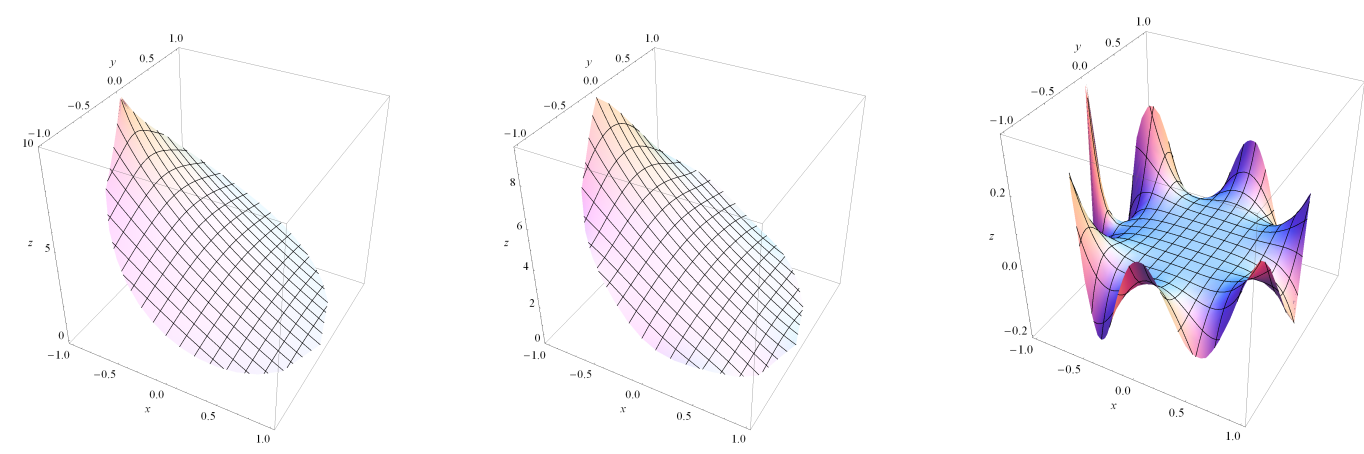

Figure 8: Example 3, $n=\ell=6$ : function $u$, interpolant $p$, error $u-p$ 


\begin{tabular}{r|l|l}
$n$ & $L_{\infty}$-error for polygon & $L_{\infty}$-error for star \\
\hline 6 & 0.725384 & 0.407932 \\
12 & 0.386227 & 0.218432 \\
24 & 0.199268 & 0.112529 \\
48 & 0.101209 & 0.057036 \\
96 & 0.0510027 & 0.0287025 \\
192 & 0.0256015 & 0.0143112 \\
384 & 0.0128259 & 0.00716828 \\
768 & 0.00641923 & 0.00358654 \\
1536 & 0.00321119 & 0.00180385 \\
3072 & 0.00160599 & 0.000874311
\end{tabular}

Table 1: Example 3: comparison of the relative $L_{\infty}$-errors for $\ell=1$ and $\ell=n$.

only $C^{0}$ on the unit circle, but analytic within the unit disk. By expanding the boundary data $g$ into its Fourier series, it can be shown that the corresponding harmonic function has the representation

$$
u(x, y)=\operatorname{Re}\left(\frac{\pi^{2}}{3}+2\left(\operatorname{Li}_{2}(-x-\mathbf{i} y)+\operatorname{Li}_{2}(-x+\mathbf{i} y)\right)\right)
$$

where

$$
\operatorname{Li}_{2}(z)=\sum_{k=1}^{\infty} \frac{z^{k}}{k^{2}}
$$

is the dilogarithm or Spence's function.

This function is approximated by a harmonic polynomial $p \in \mathcal{H}_{n}$, again comparing the convex polygon and the star cases. In Figure 8, we display the function $u$ as well as its interpolating polynomial of degree 6 (using information from 13 chords) for the case $\ell=6$, i.e., the star with the longest possible sides (cf. Figure 2, third picture), and the resulting error. In Table 1 , a comparison of the $L_{\infty}$-errors using the convex polygon $(\ell=1)$ and a star $(\ell=n)$ is presented, varying the interpolation degree $n=6 \times 2^{k}, k=1, \ldots, 10$. In both cases, the error decays linearly in $n$, and the errors for the two schemes are within a factor of less than 2 of each other.

\section{Conclusion and outlook}

We have stated an interpolation problem for harmonic functions in the unit disk given certain values of its Radon projections and have shown that this problem is uniquely solvable in the case when the Radon projections are taken along the sides of a regular polygon. Methods of computer algebra were used heavily in the proof. Finally we presented numerical examples for several possible configurations of chords and demonstrated convergence rates for functions of varying smoothness.

The formula for the matrix entries obtained by symbolic methods has served as a valuable starting point for the analytic derivation of a more general formula, allowing general choices for both $\theta$ and $t$. In future work, we plan to exploit this general result in order to show regularity of a larger family of schemes. 
For many problems, allowing the interpolation of functions satisfying an inhomogeneous partial differential equation of the form $\Delta u=f$ would be highly useful and is a possible subject of further work. Furthermore, noting the relation of the present work to the inversion of the Radon transform, a well-known ill-posed problem, problems with noisy data might prove interesting. Preliminary numerical experiments on this topic show promising results.

\section{Acknowledgments}

The authors would like to thank to Kamen Ivanov for fruitful discussions. Part of this work was completed while the first author was visiting RICAM, Austrian Academy of Sciences in Linz. The research of the first author was supported by Bulgarian National Science Fund, Grant DDVU 0230/11. The research of the second author was funded by the Austrian Science Fund (FWF): W1214-N15, project DK04. The research of the third author was funded by the FWF-project: P20162-N18. The fourth author was supported by grant P22748-N18 of the FWF. The research of the fifth author was funded by the strategic program "Innovatives OÖ 2010plus" by the Upper Austrian Government.

\section{References}

[1] B. Bojanov and C. Draganova. Surface approximation by piecewise harmonic functions. In G. Nikolov and R. Uluchev, editors, Constructive Theory of Functions, Sozopol 2010: In memory of Borislav Bojanov. Prof. Marin Drinov Academic Publishing House, Sofia, 2011.

[2] B. Bojanov and I. Georgieva. Interpolation by bivariate polynomials based on Radon projections. Studia Math., 162:141-160, 2004.

[3] B. Bojanov and G. Petrova. Numerical integration over a disc. A new Gaussian cubature formula. Numer. Math., 80:39-59, 1998.

[4] B. Bojanov and G. Petrova. Uniqueness of the Gaussian cubature for a ball. J. Approx. Theory, 104:21-44, 2000.

[5] B. Bojanov and Y. Xu. Reconstruction of a bivariate polynomial from its Radon projections. SIAM J. Math. Anal., 37:238-250, 2005.

[6] A.S. Cavaretta, C.A. Micchelli, and A. Sharma. Multivariate interpolation and the Radon transform, part I. Math. Z., 174:263-279, 1980.

[7] A.S. Cavaretta, C.A. Micchelli, and A. Sharma. Multivariate interpolation and the Radon transform, part II. In Quantitive Approximation, pages 49-62, New York, 1980. Acad. Press.

[8] M.E. Davison and F.A. Grunbaum. Tomographic reconstruction with arbitrary directions. Comm. Pure Appl. Math., 34:77-120, 1981. 
[9] I. Georgieva, C. Hofreither, and R. Uluchev. Interpolation of mixed type data by bivariate polynomials. In G. Nikolov and R. Uluchev, editors, Constructive Theory of Functions, Sozopol 2010: In memory of Borislav Bojanov. Prof. Marin Drinov Academic Publishing House, Sofia, 2011. Also available as Technical Report 2010-14 in the series of the DK Computational Mathematics Linz, https://www.dkcompmath.jku.at/publications/dk-reports/2010-12-10/view.

[10] I. Georgieva and S. Ismail. On recovering of a bivariate polynomial from its Radon projections. In B. Bojanov, editor, Constructive Theory of Functions, Varna 2005, pages 127-134. Marin Drinov Academic Publishing House, Sofia, 2006.

[11] I. Georgieva and R. Uluchev. Smoothing of Radon projections type of data by bivariate polynomials. J. Comput. Appl. Math., 215:167-181, 2008.

[12] I. Georgieva and R. Uluchev. Surface reconstruction and Lagrange basis polynomials. In I. Lirkov, S. Margenov, and J. Waśniewski, editors, Large-Scale Scientific Computing 200\%, pages 670-678, Berlin, Heidelberg, 2008. Springer-Verlag.

[13] I. Georgieva and R. Uluchev. On interpolation in the unit disk based on both Radon projections and function values. In I. Lirkov, S. Margenov, and J. Waśniewski, editors, Large-Scale Scientific Computing 2009, pages 747-755, Berlin, Heidelberg, 2010. Springer-Verlag.

[14] H. Hakopian. Multivariate divided differences and multivariate interpolation of Lagrange and Hermite type. J. Approx. Theory, 34:286-305, 1982.

[15] C. Hamaker and D.C. Solmon. The angles between the null spaces of $x$-rays. J. Math. Anal. Appl., 62:1-23, 1978.

[16] A.K. Jain. Fundamentals of Digital Image Processing. Prentice Hall, 1989.

[17] Christoph Koutschan. Advanced Applications of the Holonomic Systems Approach. PhD thesis, RISC, Johannes Kepler University, Linz, Austria, 2009.

[18] Christoph Koutschan. HolonomicFunctions (User's Guide). Technical Report 10-01, RISC Report Series, Johannes Kepler University Linz, 2010. http://www.risc.unilinz.ac.at/research/combinat/software/HolonomicFunctions/.

[19] B. Logan and L. Shepp. Optimal reconstruction of a function from its projections. Duke Math. J., 42:645-659, 1975.

[20] R. Marr. On the reconstruction of a function on a circular domain from a sampling of its line integrals. J. Math. Anal. Appl., 45:357-374, 1974.

[21] F. Natterer. The Mathematics of Computerized Tomography. Classics in Applied Mathematics 32. SIAM, 2001.

[22] G. Nikolov. Cubature formulae for the disk using Radon projections. East journal on approximations, 14:401-410, 2008.

[23] Marko Petkovšek. Hypergeometric solutions of linear recurrences with polynomial coefficients. Journal of Symbolic Computation, 14(2/3):243-264, 1992. 
[24] J. Radon. Über die Bestimmung von Funktionen durch ihre Integralwerte längs gewisser Mannigfaltigkeiten. Ber. Verch. Sächs. Akad., 69:262-277, 1917.

[25] Doron Zeilberger. A holonomic systems approach to special functions identities. Journal of Computational and Applied Mathematics, 32(3):321-368, 1990.

[26] Doron Zeilberger. The method of creative telescoping. Journal of Symbolic Computation, 11:195-204, 1991. 


\section{Technical Reports of the Doctoral Program \\ "Computational Mathematics"}

\section{1}

2011-01 S. Takacs, W. Zulehner: Convergence Analysis of Multigrid Methods with Collective Point Smoothers for Optimal Control Problems February 2011. Eds.: U. Langer, J. Schicho

2011-02 L.X.Châu Ngô: Finding rational solutions of rational systems of autonomous ODEs February 2011. Eds.: F. Winkler, P. Paule

2011-03 C.G. Raab: Using Gröbner Bases for Finding the Logarithmic Part of the Integral of Transcendental Functions February 2011. Eds.: P. Paule, V. Pillwein

2011-04 S.K. Kleiss, B. Jüttler, W. Zulehner: Enhancing Isogeometric Analysis by a Finite ElementBased Local Refinement Strategy April 2011. Eds.: U. Langer, J. Schicho

2011-05 M.T. Khan: A Type Checker for MiniMaple April 2011. Eds.: W. Schreiner, F. Winkler

2011-06 M. Kolmbauer: Existence and Uniqueness of Eddy current problems in bounded and unbounded domains May 2011. Eds.: U. Langer, V. Pillwein

2011-07 M. Kolmbauer, U. Langer: A Robust Preconditioned-MinRes-Solver for Distributed TimePeriodic Eddy Current Optimal Control Problems May 2011. Eds.: R. Ramlau, W. Zulehner

2011-08 M. Wiesinger-Widi: Sylvester Matrix and GCD for Several Univariate Polynomials May 2011. Eds.: B. Buchberger, V. Pillwein

2011-09 M. Wiesinger-Widi: Towards Computing a Gröbner Basis of a Polynomial Ideal over a Field by Using Matrix Triangularization May 2011. Eds.: B. Buchberger, V. Pillwein

2011-10 M. Kollmann, M. Kolmbauer: A preconditioned MinRes solver for time-periodic parabolic optimal control problems August 2011. Eds.: U. Langer, V. Pillwein

2011-11 C. Giannelli, B. Jüttler: Bases and dimensions of bivariate hierarchical tensor-product splines October 2011. Eds.: U. Langer, J. Schicho

2011-12 I. Georgieva, C. Hofreither, C. Koutschan, V. Pillwein, T. Thanatipanonda: Harmonic interpolation based on Radon projections along the sides of regular polygons October 2011. Eds.: U. Langer, J. Schicho

The complete list since 2009 can be found at https://www.dk-compmath.jku.at/publications/ 


\section{Doctoral Program}

\section{"Computational Mathematics"}

\section{Director:}

Prof. Dr. Peter Paule

Research Institute for Symbolic Computation

\section{Deputy Director:}

Prof. Dr. Bert Jüttler

Institute of Applied Geometry

\section{Address:}

Johannes Kepler University Linz

Doctoral Program "Computational Mathematics"

Altenbergerstr. 69

A-4040 Linz

Austria

Tel.: +443 732-2468-7174

E-Mail:

office@dk-compmath.jku.at

\section{Homepage:}

http://www.dk-compmath.jku.at 